\title{
Double-high insert presents with worse mid-term outcomes in cruciate-retaining medial-pivotal total knee arthroplasty - a propensity score matched analysis with averaged 8-year follow-up
}

Shuai Xiang

Affiliated Hospital of Qingdao University

Yingzhen Wang

Affiliated Hospital of Qingdao University

Chengyu Lv

Affiliated Hospital of Qingdao University

Changyao Wang

Affiliated Hospital of Qingdao University

Haining Zhang ( $\nabla$ hunterzhhn@126.com )

Affiliated Hospital of Qingdao University

\section{Research Article}

Keywords: total knee arthroplasty, medial-pivotal, double-high, cruciate-retaining

Posted Date: October 11th, 2021

DOl: https://doi.org/10.21203/rs.3.rs-910422/v1

License: (c) (1) This work is licensed under a Creative Commons Attribution 4.0 International License.

Read Full License 


\section{Abstract \\ Background}

The aim of this study was to compare the mid-term clinical and radiographic outcomes between medialpivotal (MP) insert and double-high (DH) insert used under cruciate-retaining condition in ADVANCE ${ }^{8}$ total knee arthroplasty (TKA).

\section{Methods}

The follow-up was conducted for 158 consecutive patients underwent unilateral ADVANCE® TKA from January 2011 to April 2014. 84 MP inserts and 74 DH inserts were used under cruciate-retaining condition. A 1:1 propensity score matching (PSM) analysis was performed between MP insert and DH insert to compare the clinical and radiographic outcomes.

\section{Results}

After a 1:1 PSM, 120 patients (60 pairs) were matched between MP insert and DH insert. The baseline demographic parameters and clinical scores were comparable between the two groups. The postoperative clinical outcomes at an averaged 8-year follow-up of both groups were significantly improved. The range of motion (ROM) of DH group was better than that of MP group and equivalent Knee Society Function Score (KSFS) between the two groups was found. However, the Knee Society Score (KSS), Western Ontario and McMaster Universities Arthritis Index (WOMAC) score and Forgotten Joint Score (FJS) of MP group were found to be significantly superior than those of DH group. Comparable complication rate and revision rate were observed between the two groups. The radiographic results were also equally good between MP and DH group.

\section{Conclusions}

Although the mid-term clinical and radiographic outcomes of DH insert are fairly good, the clinical scores of $\mathrm{DH}$ group were worse than those of MP group.

\section{Background}

Total knee arthroplasty (TKA) is the most successful procedure for patients with end-stage osteoarthritis and good to excellent long-term functional outcomes and implant survivorship has been validated [1-4]. However, the post-cam design in conventional knee implants fails to fully restore the normal motion pattern of the knee, leading to "paradoxical anterior slide" of the femur during knee flexion, which has been considered to contribute to patients' dissatisfaction [5]. Physiologically, the medial-pivotal motion 
along with femoral rollback is observed throughout the flexion motion, even in a deep flexion range [6]. To achieve better function, the medial-pivotal (MP) concept has been emphasized during implant designing.

ADVANCE ${ }^{\circledR}$ knee (MicroPort Orthopedics Inc., China) is one of the earliest MP designed implants and has been widely used in the past decade, presenting with excellent clinical outcomes and survivorship [7, 8]. During knee flexion, the medial ball-in-socket structure of the standard MP insert provides anteriorposterior stability instead of the post-cam system. Meanwhile, the arcuate groove of the lateral side allows the femoral rollback centered on the medial axis. With a congruent articular surface, the normal medial-pivotal kinematics is better restored and several studies have demonstrated decreased intercondylar stress and lessened polyethylene wear in this implant [9-12]. Despite the standard MP insert, double-high $(\mathrm{DH})$ insert is an alternative during clinical practice. The posterior lip of the $\mathrm{DH}$ insert is $3 \mathrm{~mm}$ lower than that of the standard MP insert, resulting in a posterior slope to facilitate the femoral rollback during knee flexion [13]. However, the number of research on DH insert is limited and the midterm outcomes of the DH insert are unknown compared with its MP counterpart.

In this study, we conducted an averaged 8-years' follow-up to compare the mid-term clinical and radiographic outcomes between MP insert and $\mathrm{DH}$ insert in cruciate-retaining ADVANCE ${ }^{\circ}$ TKA.

\section{Materials And Methods}

The present study was approved by the review board of our institution. The medical records of consecutive patients underwent unilateral cruciate-retaining ADVANCE ${ }^{\circledR}$ (MicroPort Orthopedics Inc., China) TKAs from January 2011 to June 2014 due to osteoarthritis were reviewed. A total of 197 patients were identified and invited to the outpatient department for the follow-up at June, 2021. 39 patients were lost to follow-up and the follow-up rate was $80.2 \%$. Among the 158 patients available for analysis, standard MP insert was used in 84 patients and $\mathrm{DH}$ insert in 74 patients.

For all patients undergoing joint replacement at our institution, preoperative functional outcomes were routinely recorded, including the range of motion (ROM), the Knee Society Score (KSS), the Knee Society Function Score (KSFS) and the Western Ontario and McMaster Universities Arthritis Index (WOMAC) score. Standing anteroposterior and lateral knee radiographs, as well as weight-bearing full-length radiograph were taken. All TKA procedures were performed by senior surgeons in our institution and pneumatic tourniquet was applied throughout the procedure. During the operation, a medial parapatellar approach was used to expose the knee and the femoral osteotomy was performed in a $5^{\circ}$ of valgus and $3^{\circ}$ of external rotation. An extramedullary guide was used to cut the proximal tibia after protecting the tibial insertion of the posterior cruciate ligament (PCL). All components were fixed with bone cement. Either DH insert or MP insert was used. Finally, a drainage tube was placed and the incision was closed. The routine postoperative care including intravenous application of cefuroxime to prevent infection, subcutaneous low-molecular-weight heparin to prevent venous thromboembolism (VTE), intravenous non-steroid anti-inflammatory drugs (NSAIDs) followed by oral administration, and daily continuous passive motion. 
The postoperative follow-up including a routine comeback to outpatient department at 6 weeks and 1 year postoperatively, as well as this final follow-up. Senior residents conducted all physical examinations and evaluated the clinical outcomes using the ROM, the KSS, the KSFS and the WOMAC score. Selfreported outcomes were also evaluated using the Forgotten Joint Score (FJS). Radiographic results were read on a PACS (General Electric, Chicago, IL, USA) monitor and measured using a mouse-point cursor and an automated computer calculation. Standing anteroposterior and lateral images at last follow-up were recorded. The femorotibial angle (FTA) and coronal position of the components, including anatomical medial proximal tibial angle (aMPTA) and anatomical lateral distal femoral angle (aLDFA) were measured. The posterior tibial slope (PTS) was also measured. The presence and location of radiolucent lines was also identified. All measurements were done using the standard method reported by Park et al[14] and Kim et al[15]. A radiolucency less than $1 \mathrm{~mm}$ was considered as a physiological radiolucent line and a pathological radiolucent line was determined as a complete radiolucency more than $1 \mathrm{~mm}$, indicating a possible implant loosening.

A 1:1 propensity score matched analysis was then performed between the patients with MP insert and DH insert, aiming to control selection bias and ensure covariate balance between the groups. The confounding variables including age, body mass index (BMI), and baseline clinical scores such as KSS, KSFS and WOMAC score were matched using propensity score calculated by logistic regression. The propensity scores and standardized differences before and after matching were calculated and showed in Figure 1.

\section{Statistical analysis}

The statistical analysis was done by SPSS 26.0 (SPSS Inc, Chicago, IL). Clinical data were presented as mean \pm standard deviation (SD). Student t-tests were used to compare the difference in continuous variables. Fisher exact test was used to determine the difference in categorical variables. $P<0.05$ was considered as statistically significant.

\section{Results}

After a 1:1 propensity score matching, the data of 120 patients (60 pairs) were analyzed. There was no statistical difference in demographic data including age (65.4 \pm 6.8 vs. $66.4 \pm 7.2, p=0.421), \mathrm{BMl}(26.9 \pm$ 3.6 vs. $27.2 \pm 3.2, p=0.600)$ and gender ratio $(20.0 \%$ vs. $10.0 \%, p=0.132)$ between $D H$ group and MP group. The length of stay (LOS, $10.7 \pm 8.4$ vs. $10.0 \pm 3.5$ days, $p=0.603)$ and follow-up period $(8.6 \pm 0.7$ vs. $8.5 \pm 1.1$ years, $p=0.812$ ) between the $\mathrm{DH}$ and MP group were also comparable (Table 1). Preoperatively, no significant difference was found in baseline WOMAC score (76.2 \pm 4.7 vs. $75.9 \pm 6.6, p=0.740)$, KSS ( $23.4 \pm 7.1$ vs. $22.8 \pm 4.8, p=0.587)$, KSFS ( $31.9 \pm 12.3$ vs. $32.6 \pm 14.4, p=0.785)$ and $R O M(82.5 \pm 16.4$ vs. $84.0 \pm 12.9, p=0.632$, Table 2 ). In both groups, significantly improved postoperative clinical outcomes were found (Table 2). At last follow-up, although the ROM of the DH group was significantly higher than that of the MP group $(111.0 \pm 10.5$ vs. $104.3 \pm 11.8, p=0.001)$, the KSS $(82.9 \pm 11.7$ vs. $90.2 \pm 5.4$, $\mathrm{p}=0.000)$, WOMAC score $(28.1 \pm 9.6$ vs. $11.6 \pm 13.4, \mathrm{p}=0.000)$, and FJS $(66.7 \pm 3.6$ vs. $77.1 \pm 24.0$, 
$p=0.000)$ were all in favor of MP group. The postoperative KSFS (73.7 \pm 12.7 vs. $73.5 \pm 12.3, p=0.942)$ was found to be equivalent between the two groups (Table 2).

Table 1

Demographics information

\begin{tabular}{|llll|}
\hline Parameters & DH group & MP group & P value \\
\hline Age (year) & $65.4 \pm 6.8$ & $66.4 \pm 7.2$ & 0.421 \\
\hline Male $(\%)$ & $20.0 \%$ & $10.0 \%$ & 0.132 \\
\hline BMI (kg/m $\left.{ }^{2}\right)$ & $26.9 \pm 3.6$ & $27.2 \pm 3.2$ & 0.600 \\
\hline Follow-up (Range, Year) & $8.6 \pm 0.7$ & $8.5 \pm 1.1$ & 0.812 \\
& $(7.0-10.2)$ & $(7.0-10.2)$ & \\
\hline Length of stay (LOS, Day) & $10.7 \pm 8.4$ & $10.0 \pm 3.5$ & 0.603 \\
\hline
\end{tabular}

Table 2

Mid-term clinical outcomes between PS group and CR group

\begin{tabular}{|llll|}
\hline Parameters & DH group & MP group & P value \\
\hline Preoperative WOMAC & $76.2 \pm 4.7$ & $75.9 \pm 6.6$ & 0.740 \\
\hline Postoperative WOMAC & $28.1 \pm 9.6$ & $11.6 \pm 13.4$ & 0.000 \\
\hline P value & 0.000 & 0.000 & \\
\hline Preoperative KSS & $23.4 \pm 7.1$ & $22.8 \pm 4.8$ & 0.587 \\
\hline Postoperative KSS & $82.9 \pm 11.7$ & $90.2 \pm 5.4$ & 0.000 \\
\hline P value & 0.000 & 0.000 & \\
\hline Preoperative KSFS & $31.9 \pm 12.3$ & $32.6 \pm 14.4$ & 0.785 \\
\hline Postoperative KSFS & $73.7 \pm 12.7$ & $73.5 \pm 12.3$ & 0.942 \\
\hline P value & 0.000 & 0.000 & \\
\hline Preoperative ROM & $82.5 \pm 16.4$ & $84.0 \pm 12.9$ & 0.632 \\
\hline Postoperative ROM & $111.0 \pm 10.5$ & $104.3 \pm 11.8$ & 0.001 \\
\hline P value & 0.000 & 0.000 & \\
\hline FJS & $66.7 \pm 3.6$ & $77.1 \pm 24.0$ & 0.000 \\
\hline
\end{tabular}

The radiographic outcomes are displayed in Table 3 and Figure 2. The preoperative FTA measured on standing anteroposterior image between $\mathrm{DH}$ and MP group was not statistically different $\left(-4.3^{\circ} \pm 6.6 \mathrm{vs}\right.$. $-4.4^{\circ} \pm 7.2, p=0.632$ ). Postoperatively, the alignment of the lower limb was corrected in both groups and 
the FTA was $3.9^{\circ} \pm 2.7$ and $3.7^{\circ} \pm 3.5$ in DH and MP group, respectively, without statistical significance $(p=0.589)$. The position of femoral component and tibial component in both groups was satisfactory, indicating by normal aLDFA $\left(83.9^{\circ} \pm 2.2\right.$ vs. $\left.84.7^{\circ} \pm 2.2, p=0.100\right)$, aMPTA $\left(88.3^{\circ} \pm 2.7\right.$ vs. $88.4^{\circ} \pm 2.3$, $p=0.767)$, and PTS $\left(4.0^{\circ} \pm 6.2\right.$ vs. $\left.4.2^{\circ} \pm 5.8, p=0.848\right)$. No pathological radiolucent line was found in both groups (Table 3).

Table 3

Radiographic Results

\begin{tabular}{|llll|}
\hline Parameters & DH group & MP group & P value \\
\hline Tibiofemoral angle (degrees) & & & \\
\hline Preoperative & $-4.3 \pm 6.6$ & $-4.3 \pm 7.2$ & 0.632 \\
\hline Final follow-up & $3.9 \pm 2.7$ & $3.7 \pm 3.5$ & 0.589 \\
\hline aLDFA (degrees) & $83.9 \pm 2.2$ & $84.7 \pm 2.2$ & 0.100 \\
\hline aMPTA (degrees) & $88.3 \pm 2.7$ & $88.4 \pm 2.3$ & 0.767 \\
\hline Posterior tibial slope & $4.0 \pm 6.2$ & $4.2 \pm 5.8$ & 0.848 \\
(degrees) & & & \\
\hline Radiolucent line $\leq 1 \mathrm{~mm}$ & $4(6.7 \%)$ & $3(5.0 \%)$ & 0.999 \\
\hline Radiolucent line $>1 \mathrm{~mm}$ & 0 & 0 & 0.999 \\
\hline
\end{tabular}

Among the 158 patients who finished the final follow-up, a total of ten complications were recorded. There were four complications in MP group (4.8\%), including two cases of continuous patellar clicking and two cases of anterior knee pain. Six complications were found in DH group (8.1\%), including two cases of continuous patellar clicking, two cases of anterior knee pain, one case of periprosthetic infection and one case of delayed healing of skin incision. The patient in suffering periprosthetic infection underwent revision TKA. The complication rate and revision rate between the two groups lack statistical significance (Table 4). 
Table 4

Overall complications

\begin{tabular}{|llll|}
\hline Complications & $\begin{array}{l}\text { DH group } \\
(\mathbf{n}=74)\end{array}$ & $\begin{array}{l}\text { MP group } \\
(\mathbf{n}=\mathbf{8 4})\end{array}$ & P value \\
\hline Infection & 1 & & \\
\hline Complications of patellofemoral joint & & & \\
\hline Continuous patellar clicking & 2 & 2 & \\
\hline Anterior knee pain & 2 & 2 & \\
\hline Delayed healing of skin incision & 1 & & 0.34 \\
\hline Total & $6(8.1 \%)$ & $4(4.8 \%)$ & 0.99 \\
\hline Revisions & $1(1.4 \%)$ & 0 & \\
\hline
\end{tabular}

\section{Discussion}

The ADVANCE $®$ knee system is one of the most widely applied medial-pivot (MP) knee implant worldwide which is characterized by the conformed medial ball-socket articulating interface [16]. This unique design to diminishes the paradoxical anterior translation of the femur and good to excellent longterm outcomes and implant survivorship have been reported [17]. Two types of tibial insert have been designed for ADVANCE® knee and can be exchanged on the same tibial tray. Compared to the conventional MP insert, the anterior and posterior lips of the DH insert are decreased by $1 \mathrm{~mm}$ and $3 \mathrm{~mm}$, respectively, forming a path for femoral rollback and aiming to achieve better flexion. However, investigation on the clinical and radiographic outcomes of $\mathrm{DH}$ insert is rare. In this study, we conducted an averaged 8-year follow-up to compare the mid-term outcomes between MP insert and DH insert through a propensity score matching analysis. With equivalent baseline parameters, although the ROM of the DH group was better, the mid-term clinical scores, including postoperative WOMAC score, KSS and FJS, of DH group were inferior to those of MP group. The KSFS between the two groups was equivalent. Our results displayed similar radiographic results between two groups. The revision rate and complication rate were also not significantly different between the two groups.

It seems that $\mathrm{DH}$ insert is not preferred by most surgeons and there has only been one published research investigating its clinical application. A comparison between MP insert and DH insert was conducted by Ishida et al. in cruciate-sacrificing TKA, including twenty knees in each group. After a mean 4-year followup, they found that the KSS, KSFS and University of California, Los Angeles (UCLA) activity score between DH group and MP group were not significantly different, although the KSS of MP group was four points higher. Meanwhile, the DH group presented with better ROM and knee flexion, but significance was still not found [18]. However, their results might be compromised by the small sample size. It is difficult to get significant results with only twenty patients in each group. In this study, we included sixty patients in each 
group through propensity score matching and provided an averaged 8-year follow-up results of DH insert, which is the longest follow-up period of DH insert. To some extent, our results partly agreed with the results of Ishida et al. Comparable KSFS and radiographic results between the two groups, superior KSS of MP group and better ROM of DH group were observed. However, compared with that of DH group, the KSS of MP group was seven points higher and the ROM was ten degrees worse, both with statistical significance. The postoperative alignment and position of the implant were measured on standing anteroposterior and lateral films. The FTA of DH group and MP group was averaged $3.9^{\circ}$ and $3.7^{\circ}$, respectively, with no statistical significance. According to previous studies, the alignment was considered as varus when the FTA was less than $2.4^{\circ}-4^{\circ}[19,15,20]$. Our results indicated that the alignment of both groups was neutral to slightly varus to maintain the medial tension, which is vital to the stability of the knee. The coronal position as well as the sagittal position of the implant was also measured and our results showed that the aLDFA, aMPTA and PTS were all equally good between the two groups. The posterior slope on $\mathrm{DH}$ insert formed between the anterior and posterior lip may further increase the overall posterior slope and thus, better ROM of DH group is reasonable. When referring to significantly worse KSS, WOMAC score and FJS of DH group, we first questioned whether this increased posterior slope of $\mathrm{DH}$ insert might cause knee instability. However, there was no patient complaining instable knee as a complication. Then we questioned that whether DH insert was suitable for cruciate-retaining procedure. In a previous research, Omori et al. specifically investigated the influence of different geometry of $\mathrm{DH}$ and MP insert on knee kinematics [13]. According to their results, under cruciate-retaining condition, similar motion pattern was observed between MP insert and DH insert and both inserts failed to reproduce medial-pivotal motion but did have bicondylar femoral rollback. However, under cruciate-sacrificing condition, although medial-pivotal kinematics was confirmed, paradoxical anterior translation of the lateral compartment was also observed from $0^{\circ}$ to $60^{\circ}$ of knee flexion, indicating that $\mathrm{DH}$ insert might not be suitable for cruciate-sacrificing procedures. Thus, we tended to believe that compared with MP insert, some minor problems might exist in the DH design, leading to worse mid-term clinical outcomes. And this might be the reason why $\mathrm{DH}$ insert is not widely used. We also admitted that the mid-term clinical outcomes of DH insert in our study is fairly good for most patients but some modifications are need to get better results.

This study provided the first report on the comparison of clinical outcomes between DH insert and MP insert in cruciate-retaining ADVANCE ${ }^{\circledR}$ TKA with the longest follow-up period and largest number of patients included. However, several limitations of this study should be acknowledged. Firstly, this was a single center study which might compromise the generalizability. Secondly, although we attempt to avoid bias through propensity score matching, the retrospective design of this study inevitably leading to bias during data analyzing. However, since 2015, DH insert was not available and cruciate-sacrificing procedure was dominantly performed using MP insert in our institution. Thus, a prospective study is not able to be carried out.

Taken together, in this averaged 8-year follow-up, we found that both MP insert and DH insert presented with good mid-term clinical outcomes in cruciate-retaining TKA, with low complication rate and revision 
rate. However, through a propensity score matching analysis, we found MP insert was superior to DH insert in clinical outcomes, including KSS, WOMAC score and FJS.

\section{Abbreviations}

MP, medial-pivotal; DH, double-high; TKA, total knee arthroplasty; PSM, propensity score matching; ROM, range of motion; KSFS, Knee Society Function Score; KSS, Knee Society Score; WOMAC, Western Ontario and McMaster Universities Arthritis Index; FJS, Forgotten Joint Score; PCL, posterior cruciate ligament; VTE, venous thromboembolism; NSAIDs, non-steroid anti-inflammatory drugs; FTA, femorotibial angle; aMPTA, anatomical medial proximal tibial angle; aLDFA, anatomical lateral distal femoral angle; PTS, posterior tibial slope.

\section{Declarations}

MP, medial-pivotal; DH, double-high; TKA, total knee arthroplasty; PSM, propensity score matching; ROM, range of motion; KSFS, Knee Society Function Score; KSS, Knee Society Score; WOMAC, Western Ontario and McMaster Universities Arthritis Index; FJS, Forgotten Joint Score; PCL, posterior cruciate ligament; VTE, venous thromboembolism; NSAIDs, non-steroid anti-inflammatory drugs; FTA, femorotibial angle; aMPTA, anatomical medial proximal tibial angle; aLDFA, anatomical lateral distal femoral angle; PTS, posterior tibial slope.

\section{References}

1. Dixon MC, Brown RR, Parsch D, Scott RD (2005) Modular fixed-bearing total knee arthroplasty with retention of the posterior cruciate ligament. A study of patients followed for a minimum of fifteen years. J Bone Joint Surg Am 87 (3):598-603. doi:10.2106/JBJS.C.00591

2. Papasoulis E, Karachalios T (2019) A 13- to 16-year clinical and radiological outcome study of the genesis II cruciate retaining total knee arthroplasty with an oxidised zirconium femoral component. Knee 26 (2):492-499. doi:10.1016/j.knee.2019.01.013

3. Kremers HM, Sierra RJ, Schleck CD, Berry DJ, Cabanela ME, Hanssen AD, Pagnano MW, Trousdale RT, Lewallen DG (2014) Comparative Survivorship of Different Tibial Designs in Primary Total Knee Arthroplasty. J Bone Joint Surg Am 96 (14):e121. doi:10.2106/JBJS.M.00820

4. Wylde V, Learmonth I, Potter A, Bettinson K, Lingard E (2008) Patient-reported outcomes after fixedversus mobile-bearing total knee replacement: a multi-centre randomised controlled trial using the Kinemax total knee replacement. J Bone Joint Surg Br 90 (9):1172-1179. doi:10.1302/0301620X.90B9.21031

5. Schmidt R, Komistek RD, Blaha JD, Penenberg BL, Maloney WJ (2003) Fluoroscopic analyses of cruciate-retaining and medial pivot knee implants. Clin Orthop Relat Res (410):139-147. doi:10.1097/01.blo.0000063565.90853.a4 
6. Nakagawa S, Kadoya Y, Todo S, Kobayashi A, Sakamoto H, Freeman MA, Yamano Y (2000)

Tibiofemoral movement 3: full flexion in the living knee studied by MRI. J Bone Joint Surg Br 82 (8):1199-1200. doi:10.1302/0301-620x.82b8.10718

7. Karachalios T, Varitimidis S, Bargiotas K, Hantes M, Roidis N, Malizos KN (2016) An 11- to 15-year clinical outcome study of the Advance Medial Pivot total knee arthroplasty: pivot knee arthroplasty. Bone Joint J 98-B (8):1050-1055. doi:10.1302/0301-620X.98B8.36208

8. Castellarin G, Pianigiani S, Innocenti B (2019) Asymmetric polyethylene inserts promote favorable kinematics and better clinical outcome compared to symmetric inserts in a mobile bearing total knee arthroplasty. Knee Surg Sports Traumatol Arthrosc 27 (4):1096-1105. doi:10.1007/s00167-0185207-9

9. Miyazaki Y, Nakamura T, Kogame K, Saito M, Yamamoto K, Suguro T (2011) Analysis of the kinematics of total knee prostheses with a medial pivot design. J Arthroplasty 26 (7):1038-1044. doi:10.1016/j.arth.2010.08.015

10. Ansari F, Ries MD, Pruitt L (2016) Effect of processing, sterilization and crosslinking on UHMWPE fatigue fracture and fatigue wear mechanisms in joint arthroplasty. J Mech Behav Biomed Mater 53:329-340. doi:10.1016/j.jmbbm.2015.08.026

11. Shu L, Yamamoto K, Kai S, Inagaki J, Sugita N (2019) Symmetrical cruciate-retaining versus medial pivot prostheses: The effect of intercondylar sagittal conformity on knee kinematics and contact mechanics. Comput Biol Med 108:101-110. doi:10.1016/j.compbiomed.2019.03.005

12. Minoda Y, Kobayashi A, Iwaki H, Miyaguchi M, Kadoya Y, Ohashi H, Yamano Y, Takaoka K (2003) Polyethylene wear particles in synovial fluid after total knee arthroplasty. Clin Orthop Relat Res (410):165-172. doi:10.1097/01.blo.0000063122.39522.c2

13. Omori G, Onda N, Shimura M, Hayashi T, Sato T, Koga Y (2009) The effect of geometry of the tibial polyethylene insert on the tibiofemoral contact kinematics in Advance Medial Pivot total knee arthroplasty. J Orthop Sci 14 (6):754-760. doi:10.1007/s00776-009-1402-3

14. Park A, Stambough JB, Nunley RM, Barrack RL, Nam D (2016) The Inadequacy of Short Knee Radiographs in Evaluating Coronal Alignment After Total Knee Arthroplasty. J Arthroplasty 31 (4):878-882. doi:10.1016/j.arth.2015.08.015

15. Kim YH, Park JW, Kim JS, Park SD (2014) The relationship between the survival of total knee arthroplasty and postoperative coronal, sagittal and rotational alignment of knee prosthesis. Int Orthop 38 (2):379-385. doi:10.1007/s00264-013-2097-9

16. Cho SH, Cho HL, Lee SH, Jin HK (2013) Posterior femoral translation in medial pivot total knee arthroplasty of posterior cruciate ligament retaining type. J Orthop 10 (2):74-78. doi:10.1016/j.jor.2013.04.004

17. Fitch DA, Sedacki K, Yang Y (2014) Mid- to long-term outcomes of a medial-pivot system for primary total knee replacement: a systematic review and meta-analysis. Bone Joint Res 3 (10):297-304. doi:10.1302/2046-3758.310.2000290 
18. Ishida K, Matsumoto T, Tsumura N, Iwakura T, Kubo S, Iguchi T, Akisue T, Nishida K, Kurosaka M, Kuroda R (2014) No difference between double-high insert and medial-pivot insert in TKA. Knee Surg Sports Traumatol Arthrosc 22 (3):576-580. doi:10.1007/s00167-012-2314-x

19. Ritter MA, Davis KE, Meding JB, Pierson JL, Berend ME, Malinzak RA (2011) The effect of alignment and BMI on failure of total knee replacement. J Bone Joint Surg Am 93 (17):1588-1596. doi:10.2106/JBJS.J.00772

20. Morgan SS, Bonshahi A, Pradhan N, Gregory A, Gambhir A, Porter ML (2008) The influence of postoperative coronal alignment on revision surgery in total knee arthroplasty. Int Orthop 32 (5):639642. doi:10.1007/s00264-007-0391-0

\section{Figures}




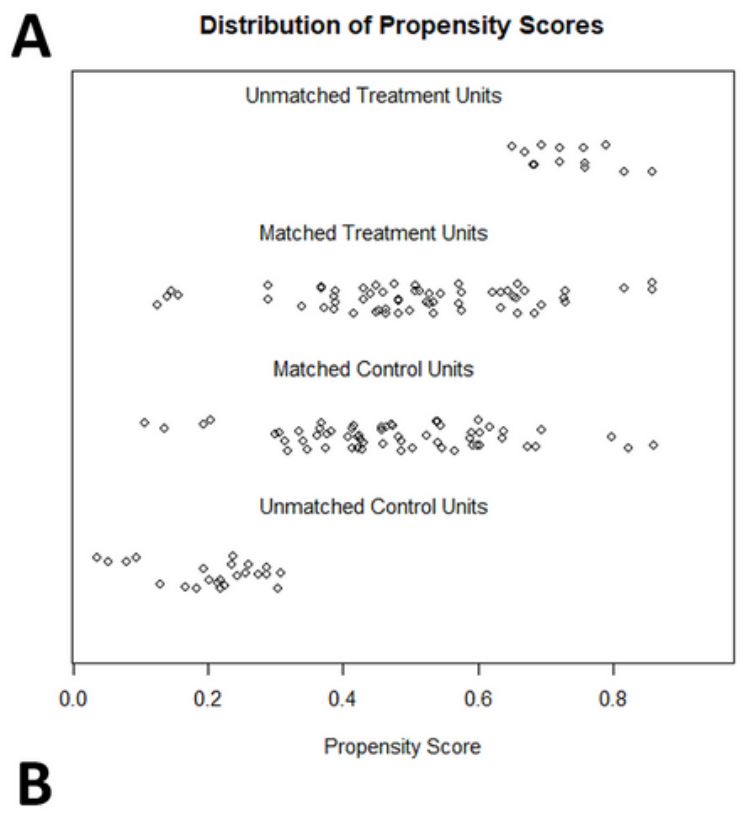

Standardized differences before matchin!
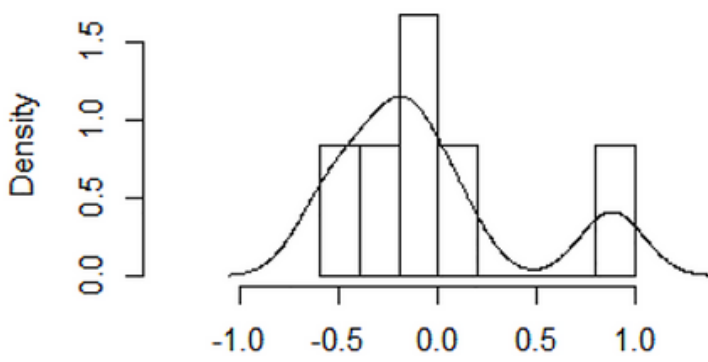

Std. difference

\section{Standardized differences after matching}
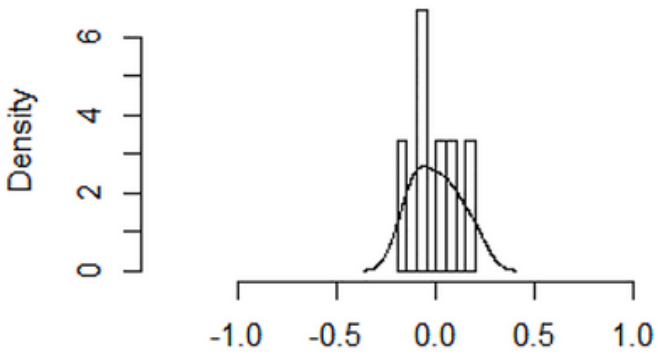

Std. difference

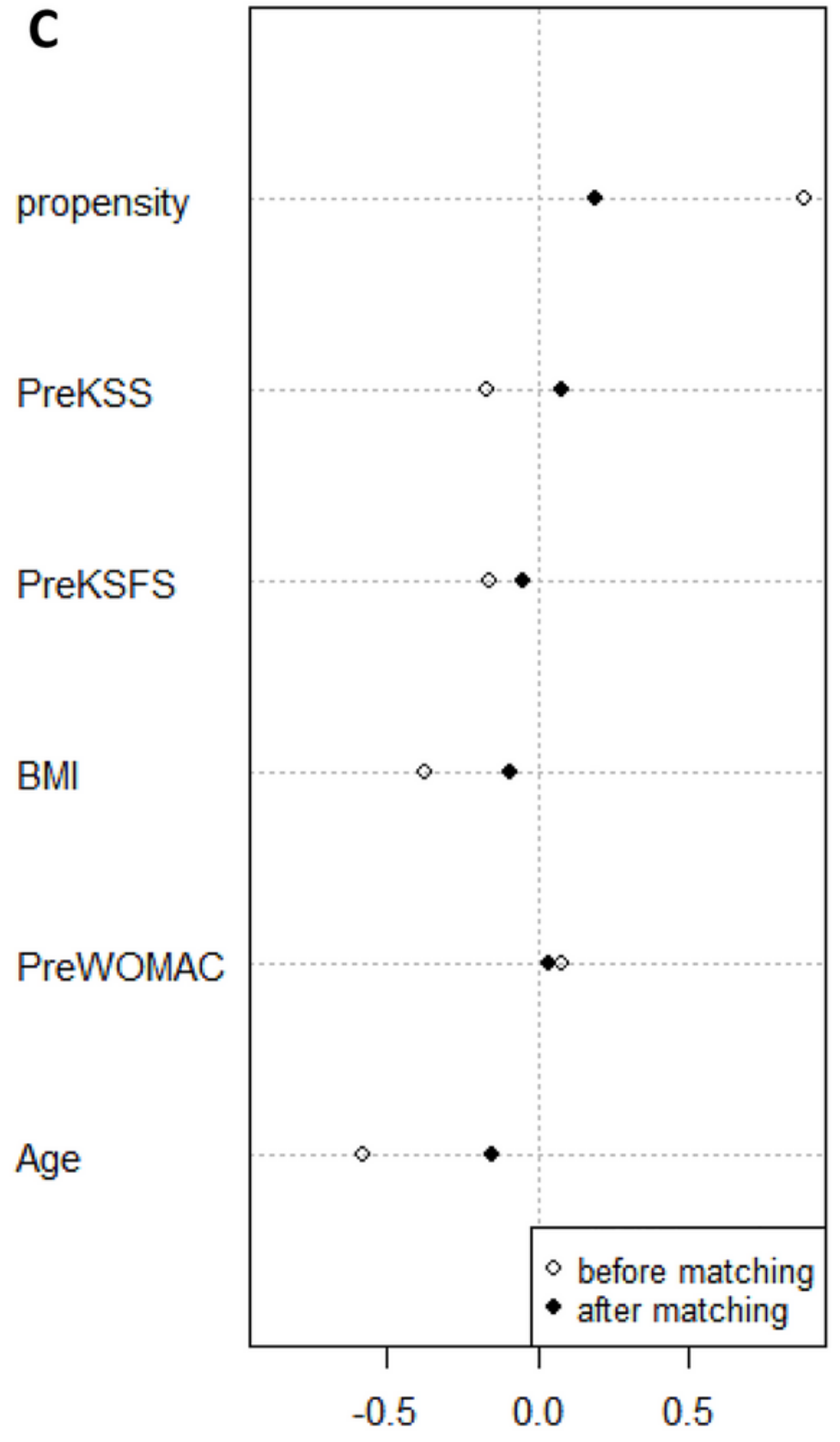

\section{Figure 1}

Results of propensity score matching. (A) Density plots after propensity score matching. (B) Standarized differences before and after propensity score matching. (C) Confounding variables before and after propensity score matching. 
Figure 2

Preoperative and final X-ray of medial-pivotal (MP) insert (panel A-D) and double-high (DH) insert (panel $\mathrm{E}-\mathrm{H})$ used in cruciate-retaining TKA. 